\title{
Topographies lacking tidal conversion
}

\author{
Leo R. M. Maas \\ NIOZ Royal Netherlands Institute for Sea Research, PO Box 59, 1790 AB Texel. The Netherlands \\ Institute for Marine and Atmospheric Research Utrecht. Utrecht University. Princetonplein 5. 3584 CC. \\ The Netherlands
}

(Received 10 March 2011: revised 10 March 2011: accepted 27 May 2011: first published online 2 August 2011)

The consensus is that in a stratified sea a classical model of tidal flow over irregular but smooth topography necessarily leads to the generation of internal tides, regardless of the shape of the topography. This is referred to as tidal conversion. Here it is shown, however, that there exists a large class of topographies for which there is neither tidal conversion nor any scattering of incident internal waves. This result is obtained in a uniformly stratified, rigid-lid sea using a barotropic tide that, owing to its large horizontal scale, is supposed to be simply a mass-conserving, periodic back-and-forth flow. The baroclinic response at the tidal frequency is, upon non-dimensionalizing and stretching of coordinates, determined by a standard hyperbolic boundary value problem (BVP). We here solve this hyperbolic BVP by mapping a domain of complicated, yet a priori unknown shape, onto a uniform-depth channel for which the same hyperbolic problem is known to display neither conversion of the barotropic tide nor scattering of internal wave modes. The map achieving this is required to satisfy hyperbolic Cauchy-Riemann equations, defined as analogues of the Cauchy-Riemann equations that are used in solving elliptic problems. Mapping the rigid-lid surface in the original Cartesian frame onto a rigid-lid surface in the transformed frame, this map is solved in terms of one arbitrary function. Each particular function defines a new topographic shape that can be computed a posteriori. The map is unique provided the Jacobian of transformation does not vanish, which is guaranteed for subcritical bottom topography, whose slope is everywhere less than that of the characteristics. For topographies that can thus be mapped onto a channel, tidal conversion and scattering are absent. Examples discussed include the (classical) wedge, a (near-Gaussian) ridge, a continental slope and (near) sinusoidal topographies.

Key words: internal waves, wave scattering, wave-structure interactions

\section{Introduction}

It is commonly believed that in a stratified sea, tidal motion over uneven topography necessarily leads to the generation of internal tides (St Laurent et al. 2003). This is, however, not always true, as evidenced by the example in figure 1. This displays the baroclinic streamfunction produced by a barotropic flow over a ridge in a uniformly stratified, rigid-lid fluid, neatly vanishing at the bottom and surface. It just evokes a trapped response over the ridge, lacking internal tide radiation. The precise circumstances under which this solution is computed will be discussed below, but 


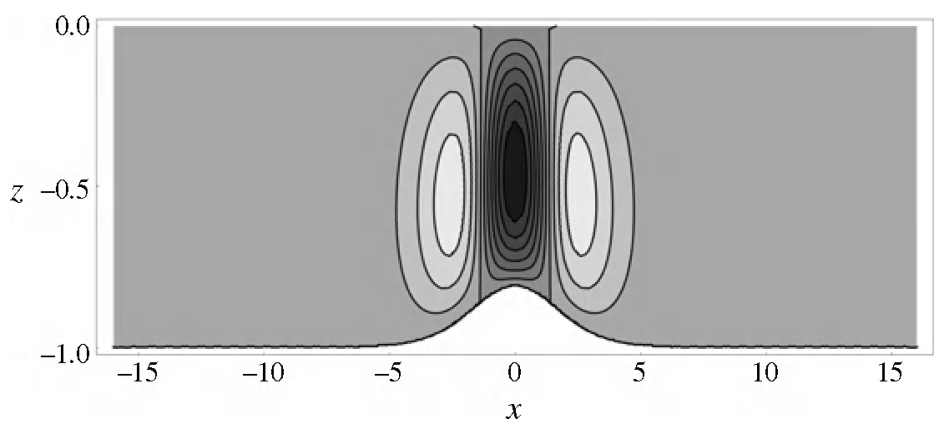

FIGURE 1. Baroclinic streamfunction field over a ridge. Details of its derivation are discussed below.

this result should not come as too big a surprise. It is reminiscent of the response in similar circumstances, obtained by Pétrélis, Llewellyn Smith \& Young (2006), who found that tidal conversion may indeed be absent over a triangularly shaped ridge due to destructive interference. This occurs when internal wave characteristics exactly span the ridge in an even number of bounces. A similar result was obtained in Baines (1973) over a piecewise linear topography, connecting a deep flat ocean to a flat shelf. The disappearance of conversion was considered something of a curiosity. For the topographies of infinitesimal height that Pétrélis et al. (2006) originally considered, this occurs at parameter values which they termed 'null points' of their parameter space. Their occurrence appeared to rely on the finite width of the triangular ridge as it could then be argued that rightward-propagating waves, forced on the left side of the ridge, could be exactly annihilated by rightward-propagating waves, following the same characteristic path, that were forced on the right side of the ridge. Note that this does not preclude the set-up of a localized response over the ridge - wave localization, in the terminology of Griffiths \& Grimshaw (2007) - produced by constructive interference of waves generated at the right side, but propagating leftward. However, the absence of tidal conversion, as found for the continuous topography of figure 1, may be a much more common phenomenon. Here we will show that there exists a whole family of topographies that actually lack conversion of the surface tide. The topographies considered are smooth, either have an infinite or finite support, and are subcritical, i.e. have a slope that is everywhere less than that of the internal wave characteristics.

The absence of energy transfer between the barotropic tide and internal tides has a counterpart in the absence of scattering of incident internal wave modes for topographies that meet the same 'resonance' criteria (Sandstrom 1976; Müller \& Liu 2000; Bühler \& Holmes Cerfon 2011). This strongly suggests that these two features are related. Indeed, as we will show, topographies for which tidal conversion is absent also lack scattering of incident internal tidal modes into other modes.

The paper is organized as follows. In $\S 2$ we discuss a classical two-dimensional internal tide generation model which also describes internal wave scattering problems. We employ a very simple and commonly used surface tide model, in which the surface is treated as rigid and the tide as a simple back-and-forth flow. In $\S 3$ we introduce a canonical map, that maps channels of complicated shapes onto those of simple shape while leaving the governing equation and boundary conditions unaffected. This occurs provided the map is of particular type and provided bottom slopes are subcritical. In $\S 4$ we discuss four examples of this map. It is applied to a wedge-shaped basin, first considered by Wunsch (1968), an isolated near-Gaussian ridge, a slope-shelf 
topography, and, finally, a near-sinusoidal periodic bottom. Using a piecewise defined map, the last case can be constrained to cover just a single near-sinusoid that is smoothly connected to an otherwise constant depth sea, effectively again building a ridge but now of finite support. Due to the presence of an arbitrary function, defining the map, an infinite amount of other topographies can be constructed for which tidal conversion and scattering are absent. Recurrent application of these maps allows the construction of still more convoluted shapes of topography lacking tidal conversion. The appearance of many conversion-free topographies raises questions concerning possible limitations involved in the simplified nature of the adopted barotropic tidal model, and concerning the relevance of the presence of a conversion-free topography closely resembling topographic shapes that do generate internal tides, addressed in the concluding $\S 5$.

\section{Tidal conversion and scattering}

In a two-dimensional, uniformly stratified fluid, the spatial structure of the stationary streamfunction field $\psi(x, z)$, produced by time $(t)$ periodic tidal flow over topography, $\alpha \exp (-\mathrm{i} t)$, is described by a spatial hyperbolic wave equation (see Appendix),

$$
\psi_{x x}-\psi_{z z}=0 .
$$

Here, $x$ and $z$ denote non-dimensional and stretched horizontal and vertical Cartesian coordinates respectively and the subscript-derivative convention is employed. This equation has to be solved subject to the condition that the rigid-lid surface, $z=0$, and bottom, $z=H(x)<0$, are streamlines. Here, non-dimensional depth, $H(x)=\hat{H}(x) d$, is the product of a dimensionless bottom-shape, $\hat{H}(x)<0$, and a depth scale, $d$. In the analysis presented here the shape of the bottom is made explicit only a posteriori. The depth scale is an inverse Froude number, defined as

$$
d=\left(\frac{N^{2}}{\omega^{2}}-1\right)^{1 / 2} \frac{D}{L}
$$

where $N$ and $\omega$ denote the stability and wave frequencies respectively, and $D$ and $L$ are representative vertical and horizontal scales of the fluid domain. Because of its lumped character (Maas \& Lam 1995), many different combinations of aspect ratio, $D / L$, and frequency ratio, $N / \omega$, all give rise to the same depth scale $d$, which is the only quantity that will be determined here. The boundary conditions for tidal flow over topography in a stratified sea thus read: $\psi=0$ at $z=0$, and $\psi=Q$ at $z=H(x)$. We define the barotropic tide as that part of the flow which carries the vertically integrated mass transport. For the tidal conversion problem, we scale the streamfunction by this barotropic tidal transport (see Appendix), in which case $Q=1$. When considering the scattering of incident internal waves, $Q=0$, since these waves carry no net transport.

In the rigid-lid model, the presence of the barotropic tide manifests itself only in a non-zero transport value $(\psi=1)$ at the bottom, which, however, is uniform along the bottom. Apart from its application at a bottom of non-constant depth, spatial localization of the internal tide forcing is therefore not really evident from this boundary condition. Note, by contrast, that localization of the forcing (to sloping regions of the topography) does become evident in the baroclinic problem that is obtained when a frequently used split is made:

$$
\psi=\bar{\psi}+\psi^{\prime} .
$$


Here, $\bar{\psi}=z / H(x)$ is called the hydrostatic barotropic tide, since the associated horizontal velocity, $-\bar{\psi}_{z}$, is uniform with depth. In that case $\psi^{\prime}$ contains the baroclinic as well as non-hydrostatic barotropic tide. Then, the boundary value problem for this field defines itself as (Baines 1982; Vlasenko, Stashchuk \& Hutter 2005; Garrett \& Gerkema 2007)

$$
\psi_{x x}^{\prime}-\psi_{z z}^{\prime}=-z\left(\frac{1}{H}\right)_{x x},
$$

subject to the boundary condition $\psi^{\prime}=0$ at both surface, $z=0$, and bottom, $z=H(x)$. Evidently, the body force on the right-hand side of (2.3) is non-zero only above sloping or curved regions, where $H_{x}$ or $H_{x x} \neq 0$.

A relatively new method to solve this problem over topographies that, far away from the bottom irregularity $(|x| \rightarrow \infty)$, revert to one and the same depth, $H(x) \rightarrow$ $h \equiv-d$, is called the boundary integral or Green's function method (Balmforth, Ierley \& Young 2002; Llewellyn Smith \& Young 2002). It writes $\psi=\psi_{c}+\tilde{\psi}$, using a known solution, $\psi_{c}$, for a comparison topography, $H_{c}(x)$. This is usually a straight channel of constant depth, equal to the asymptotic depth of the actual topography, $H_{c}=h$. For such a channel, $\psi_{c}=z / h$ solves the comparison BVP. Then, inserting the irregular bottom, $H(x), \psi_{c}$. would produce a flow through the actual bottom, because $\psi_{c}(x, H(x))=H(x) / h \neq 1$. By judiciously choosing a second part of the streamfunction, $\tilde{\psi}$, satisfying the wave equation but annihilating the flow at the comparison topography, $\tilde{\psi}(x, H(x))=1-H(x) / h$, vanishing of the forcing at the boundary of the $\tilde{\psi}$-field, far from its irregular region,

$$
\lim _{|x| \rightarrow \infty} \tilde{\psi}(x, H(x)) \rightarrow 0
$$

can be guaranteed. The $\bar{\psi}$-field contains in principle both a barotropic and a baroclinic part. The former is simply present because the comparison topography $H_{c}(x)$ does not represent the actual topography $H(x)$, but it is evident that the barotropic part of $\tilde{\psi}$ must be localized in view of (2.4). For causality reasons, $\tilde{\psi}$ needs to satisfy a radiation condition, stating that baroclinic waves only propagate away from the bottom irregularity. The method first assumes that the bottom differs from the comparison topography in a single point only (producing a Dirac delta source). By convolving the Green's function solution thus obtained with the actual bottom shape, the complete solution is obtained.

Here we use an alternative method, analogous to conformal mapping. In the context of surface wave problems, Howard \& Yu (2007) and Yu \& Howard (2010) successfully employed conformal mapping in solving the Laplace equation and boundary conditions in a rectangular channel with periodic corrugation. The internal tide generation or internal wave scattering problems are, however, hyperbolic in nature, whence the need for a hyperbolic counterpart to conformal mapping. As the boundary conditions in the hyperbolic problem are simpler than those for the surface wave problem, in this case a more general class of boundary shapes proves exactly solvable.

\section{Mapping the wave equation}

Using the general coordinate transform

$$
(x, z) \rightarrow(\xi, \zeta),
$$


by repeated use of the chain rule, $\psi_{x}=\psi_{\xi} \xi_{x}+\psi_{\zeta} \zeta_{x}$, and similarly for the $z$-derivative, the hyperbolic wave equation (2.1) transforms to

$$
\begin{aligned}
& \psi_{\xi \xi}\left(\xi_{x}^{2}-\xi_{z}^{2}\right)+2 \psi_{\xi \zeta}\left(\xi_{x} \zeta_{x}-\xi_{z} \zeta_{z}\right)+\psi_{\zeta \zeta}\left(\zeta_{x}^{2}-\zeta_{z}^{2}\right) \\
& \quad+\psi_{\xi}\left(\xi_{x x}-\xi_{z z}\right)+\psi_{\zeta}\left(\zeta_{x x}-\zeta_{z z}\right)=0
\end{aligned}
$$

In general this equation is quite complicated, but by choosing the coordinate transform to obey hyperbolic Cauchy-Riemann equations (see Appendix),

$$
\begin{aligned}
& \xi_{x}=\zeta_{z}, \\
& \xi_{z}=\zeta_{x},
\end{aligned}
$$

equation (3.2) simplifies considerably. Not only do the coefficients of the single derivative terms vanish, because both $\xi$ and $\zeta$ satisfy a hyperbolic wave equation, (2.1), but so does the coefficient multiplying the cross-derivative term, $\psi_{\xi \zeta}$. The coefficients multiplying the remaining second-derivative terms are equal, except for a sign change, and are identical to the Jacobian of transformation

$$
J \equiv J(\xi, \zeta)=\xi_{x} \zeta_{z}-\xi_{z} \zeta_{x}
$$

The transformed equation therefore reduces to

$$
J\left(\psi_{\xi \xi}-\psi_{\zeta \zeta}\right)=0 .
$$

In other words, provided Jacobian $J$ does not vanish in the new coordinate frame inside the fluid domain, $J \neq 0$, the streamfunction field satisfies exactly the same (hyperbolic wave) equation as in the original coordinate frame

$$
\psi_{\xi \xi}-\psi_{\zeta \zeta}=0
$$

The advantage of employing the transformation is that one can map the original fluid domain $H(x) \leqslant z \leqslant 0$ onto a fluid domain $h(\xi) \leqslant \zeta \leqslant 0$, for which one does have explicit solutions. Here we will restrict ourselves to a transformed fluid domain that is a constant-depth channel, $h=$ constant. This depth is, except for a sign change, taken equal to the dimensionless depth scale, $h=-d$, so that shape function $\hat{H}(x)$ can be inferred once $H(x)$ has been determined. For such a channel, the solution of (3.7), satisfying $\psi=0$ at $\zeta=0$ and $\psi=1$ at $\zeta=h$, reads

$$
\psi=\frac{\zeta}{h}+\sum_{n=-\infty}^{\infty} \hat{\psi}_{n} \sin \left(n \pi \frac{\zeta}{h}\right) \exp \left[\mathrm{i} n \pi \frac{\xi}{h}\right] .
$$

This solution is obtained by separation of variables and consists of a particular solution (first term), satisfying the bottom boundary condition, and a homogeneous solution (series expression), that vanishes at both boundaries for integer $n$. The former is the barotropic part, representing an oscillatory motion of the fluid, and the latter the baroclinic part, representing vertically standing, horizontally propagating internal wave modes of amplitude $\hat{\psi}_{n}$. Note that the zeroth-order term $(n=0)$ of the baroclinic part vanishes. Taking the suppressed time-dependence $\propto \exp (-\mathrm{i} t)$ into account and recalling that $h<0$, the terms in this series represent waves propagating from right to left $(n>0)$ or vice versa $(n<0)$. Amplitude $\hat{\psi}_{ \pm n}$ represents the strength of the $n$th incident internal wave mode at the far right or left respectively. Normally, conversion (Baines 1973; Sandstrom 1976; Craig 1987; Llewellyn Smith \& Young 2002; Vlasenko et al. 2005) or scattering (Müller \& Liu 2000; Bühler \& Holmes Cerfon 2011) problems revolve on finding those particular distributions of amplitudes for which a radiation condition 
is satisfied at infinity. For this, it is required that, to the right of a scattering region, the time-averaged, $\langle\cdot\rangle=(2 \pi)^{-1} \int_{i n}^{2 \pi} \cdot \mathrm{d} t$, vertically integrated horizontal energy flux is positive,

$$
\int_{h}^{0}\langle p u\rangle \mathrm{d} z \geqslant 0,
$$

i.e. directed outwards, to the right, and vice versa to the left of the scattering region (where $u$ represents the horizontal velocity). However, here we will derive baroclinic solutions, vanishing at bottom and surface, that do not propagate away at all. These solutions have pressure and velocity (streamfunction) fields that are exactly out-of-phase, such that the above integral vanishes. Apart from its use in $\S 4.1$, in the rest of the paper, and in particular in the tidal conversion problem, this 'baroclinic' field needs to be understood as the non-hydrostatic barotropic part, even though it has a vertically non-uniform horizontal velocity field associated with it (Garrett \& Gerkema 2007). It distinguishes itself from true internal waves in pertaining only to a non-propagating, trapped response.

Since $\xi$ and $\zeta$ obey the hyperbolic Cauchy-Riemann equations, and thus satisfy the hyperbolic wave equation (2.1), their general expressions read

$$
\begin{aligned}
& \xi=f(x+z)+g(x-z), \\
& \zeta=f(x+z)-g(x-z),
\end{aligned}
$$

where $f$ and $g$ are arbitrary functions of their argument. But, since we want the rigid-lid surface in the original frame, $z=0$, to be mapped onto the rigid lid in the transformed frame, $\zeta=0$, we choose $g(q)=f(q)$. Thus, the transformed variables are here taken to satisfy

$$
\begin{aligned}
& \xi=f(x+z)+f(x-z), \\
& \zeta=f(x+z)-f(x-z),
\end{aligned}
$$

where $f(q)$ itself is absolutely arbitrary, apart from differentiability restrictions $\left(f_{q} \neq\right.$ 0 and $f_{q q} \neq 0$ ), imposed by requiring the Jacobian to be non-vanishing (see next subsection), and by requiring that (3.12) and (3.13) satisfy the wave equation (2.1), employed in simplifying (3.2).

\subsection{Bottom shape}

Up to now, the particular shape of the bottom, $H(x)$, has been left unspecified. We can compute it a posteriori once we have made a choice for the arbitrary function $f(q)$, but we have no a priori control over it: not every function $H(x)$ can be determined by an $f(q)$. It may not be what we want, but this is what we get using this solution method.

Since we map the bottom boundary onto a constant-depth coordinate line, $\zeta=h$, the implicit description of the bottom in the original coordinate frame reads

$$
h=f(x+z)-f(x-z) .
$$

Its explicit form can formally be cast as $z=H(x)$ or $x=\bar{H}(z)$. While it depends on the particular shape of $f(q)$ whether this can be expressed analytically or not, this is not crucial, since the implicit bottom description applies anyway. Unfortunately, there is no general way of anticipating which functions $f(q)$ will lead to nice analytic solutions for $H(x)$, but the next section will illustrate the topographic shapes implied by this relation for several particular choices of $f(q)$. 


\subsection{Non-vanishing Jacobian}

One point of concern in mapping the wave equation from the Cartesian to the transformed plane is the requirement that Jacobian $J$, (3.5), is non-vanishing. Using (3.4), this Jacobian can be rewritten as

$$
J=\xi_{x}^{2}-\xi_{z}^{2}=\left(\xi_{x}-\xi_{z}\right)\left(\xi_{x}+\xi_{z}\right)=(\xi-\zeta)_{x}(\xi+\zeta)_{x} .
$$

But, using (3.12) and (3.13), this can be written as

$$
J=4 f^{\prime}(x-z) f^{\prime}(x+z) .
$$

where a prime indicates a derivative to its argument. Therefore, when a particular function $f(q)$ is chosen, non-vanishing of the Jacobian is guaranteed whenever $f^{\prime}(q) \neq 0$ for all arguments $q$.

This restriction also has a physical interpretation. To see this, take the $x$-derivative of the implicit relation defining the bottom, (3.14), applied at $z=H(x)$. This yields

$$
H^{\prime}(x)=\frac{f^{\prime}(x-H)-f^{\prime}(x+H)}{f^{\prime}(x-H)+f^{\prime}(x+H)},
$$

where a prime again indicates a derivative to its argument. The bottom slope is critical, i.e. has the same slope as the characteristic, whenever $H^{\prime}(x)= \pm 1$. This occurs for $f^{\prime}(q)=0$ or $f^{\prime}(q)= \pm \infty$, when either $q=x \pm H(x)$. Hence, vanishing of the derivative of $f(q)$ shows the breakdown of the transformation by the bottom developing a critical slope somewhere.

\section{Examples}

\subsection{The wedge: $f(q)=\ln q$}

Choosing $f(q)=\ln q$, the transformed coordinates, (3.12) and (3.13), read

$$
\begin{aligned}
& \xi=\ln (x+z)+\ln (x-z)=\ln \left(x^{2}-z^{2}\right), \\
& \zeta=\ln (x+z)-\ln (x-z)=\ln \left[\frac{x+z}{x-z}\right] .
\end{aligned}
$$

Verify that this maps the surface $z=0$ onto $\zeta=0$. The bottom shape $z=H(x)$ is determined by (3.14) and is mapped to a constant depth $\zeta=h$, so that it follows from

$$
h=\ln \left[\frac{x+z}{x-z}\right] \text {. }
$$

This is solved by

$$
z=H(x)=s x, \quad x>0,
$$

where slope

$$
s=\tanh \frac{h}{2} .
$$

In view of the employed scaling of the horizontal and vertical coordinates, $s=$ $s_{*}\left(N^{2} / \omega^{2}-1\right)^{1 / 2}$ is the ratio of the actual bottom slope, $s_{*}$, and the slope of the characteristics along which internal wave energy propagates, $\left(N^{2} / \omega^{2}-1\right)^{-1 / 2}$. For any channel depth in the transformed plane $-\infty<h<0$, this leads to a slope $-1<s<0$. Thus this covers subcritically sloping wedges only. Equation (3.16) shows that the 
Jacobian $J=4 /\left(x^{2}-z^{2}\right)=4 \mathrm{e}^{-\xi}$ never vanishes. The critical lines $z= \pm x$ are outside the fluid domain.

Remembering that $h<0$, the internal wave solutions, restricted to waves propagating leftwards, towards the apex $(x, z)=(0,0)$, i.e. $(\xi \rightarrow-\infty, \zeta=0)$, read, for $n \in \mathbb{N}$ :

$$
\psi(\xi, \zeta)=\sin \left(\frac{n \pi \zeta}{h}\right) \exp \left[\mathrm{i}\left(\frac{n \pi \xi}{h}\right)\right] .
$$

These were first studied by Wunsch (1968) and have since been applied in many internal wave studies (Wunsch 1971; Ou \& Maas 1986; Gerkema 2011, for instance). Note that (4.6) contains solutions $\operatorname{xexp}[\mathrm{i}(n \pi(\xi \pm \zeta) / h)]$ which, by (4.1) and (4.2), are proportional to functions of $x \pm z$ respectively, that obviously obey the wave equation. Inserting (4.1) and (4.2) into (4.6), and incorporating the oscillatory time dependence, now yields the explicit solution for the $n$th internal wave mode $(n \in \mathbb{N})$

$$
\psi=\sin \left(\frac{n \pi}{h} \ln \left[\frac{x+z}{x-z}\right]\right) \exp \left[\mathrm{i}\left(\frac{n \pi}{h} \ln \left(x^{2}-z^{2}\right)-t\right)\right] .
$$

Inserting the dimensional quantities for $x$ and $z$ yields Wunsch's original expression. For $n>0$ this displays the wedge as an internal wave point attractor. Geometrically, this 'attraction' is obvious since the slope of the characteristics $( \pm 1)$ is always greater than that of the bottom, so that the waves do not change their horizontal propagation direction. While the streamfunction is bounded everywhere, this does not prohibit the velocity magnitude from blowing up at the approach of the apex. In reality, this must evoke some wave-absorbing mechanism (such as viscous damping or wave breaking). Note that an expression for the pressure field follows from the hyperbolic Cauchy-Riemann equations that streamfunction and pressure satisfy, (A9), and thus reads

$$
p=-\mathrm{i} \cos \left(\frac{n \pi}{h} \ln \left[\frac{x+z}{x-z}\right]\right) \exp \left[\mathrm{i}\left(\frac{n \pi}{h} \ln \left(x^{2}-z^{2}\right)-t\right)\right] .
$$

The $\mathrm{i}$ in the pressure's amplitude is multiplied by a similar factor in the scaling (see Appendix), so that pressure and streamfunction are in-phase (actually, $180^{\circ}$ out-of-phase), representing a non-vanishing energy flux accompanying the incident wave, directed into the apex.

In addition to the internal wave modes, the wave equation also allows for a barotropic streamfunction solution in the wedge (first term of (3.8)),

$$
\psi=\frac{\zeta}{h} \mathrm{e}^{-\mathrm{i} t}=\frac{1}{h} \ln \left[\frac{x+z}{x-z}\right] \mathrm{e}^{-\mathrm{i} t} .
$$

For this we need to exclude the apex so as to avoid multiply defined streamfunction values. The corresponding pressure field is

$$
p=\frac{\xi}{h} \mathrm{e}^{-\mathrm{i} t}=\frac{1}{h} \ln \left(x^{2}-z^{2}\right) \mathrm{e}^{-\mathrm{i} t} .
$$

Factor $\mathrm{i}$ in the pressure's scaling indicates this field $(\alpha \sin t)$ is in quadrature with the streamfunction field $(\alpha \cos t)$, and conversion of the barotropic tide is absent. The spatial part of the streamfunction field is still bounded, $0 \leqslant \psi \leqslant 1$, but, as for the baroclinic solution, the corresponding velocity field blows up on approaching the apex. The rudimentary way in which a rigid-lid, stratified fluid accommodates the rise and fall of the tide with its unresolved moving shoreline by storing unphysical effects 
in positive and negative singularities in the apex exploits the fact that, paraphrasing Richard Feynman, 'there is plenty of room at infinity...'.

Wunsch's (1968) modal internal wave construction in a wedge is one of the few cases for which exact solutions for the internal wave scattering problem were obtained in a non-trivially shaped geometry (with $Q=0$ at bottom, see $\S 2$ ). (For a recent exact, analytic internal wave field in a trapezoid, containing a wave attractor, see Maas (2009).) It now transpires that the wedge furnishes just the first member of an infinite sequence of geometries for which internal and barotropic wave solutions can be found. We therefore continue by discussing a few examples explicitly.

$$
\text { 4.2. Ridge: } f(q)=[q+\delta \tanh (q / 2)] / 2
$$

Taking

$$
f(q)=[q+\delta \tanh (q / 2)] / 2 .
$$

we obtain as new coordinates

$$
\begin{aligned}
& \xi(x, z)=x+\delta \frac{\sinh x}{\cosh z+\cosh x}, \\
& \zeta(x, z)=z+\delta \frac{\sinh z}{\cosh z+\cosh x} .
\end{aligned}
$$

By construction, $z=0$ again maps onto $\zeta=0$. But topography $z=H(x)$, that maps onto a channel of fixed depth $h$, is obtained by solving $\zeta=h$, or

$$
h=z+\delta \frac{\sinh z}{\cosh z+\cosh x} .
$$

This shows that for $x \rightarrow \pm \infty$, bottom $z=H(x) \rightarrow h=-d$. Since $H \equiv \hat{H}(x) d$, with $d$ the depth scale, the shape function has the following asymptote: $\lim _{x \rightarrow \pm \infty} \hat{H}(x) \rightarrow-1$. At $x=0$, the height of the ridge, $z(h, \delta)$, follows from inverting $h=z+\delta \tanh (z / 2)$. Parameter $\delta$ is proportional to the height of the ridge above the ocean bottom. This can be seen explicitly when $|h| \ll 1$, as one finds $\hat{H}(0) \approx-1 /(1+\delta / 2)$ and thus a (dimensionless) ridge height: $\hat{H}(0)-\hat{H}( \pm \infty) \approx \delta /(2+\delta)$.

In fact, (4.14) yields the topography explicitly in inverse form, $x=\bar{H}(z)$, whose shape is

$$
\bar{H}(z)= \pm \operatorname{arccosh}\left(\frac{\delta}{h-z} \sinh z-\cosh z\right) .
$$

This topography is close to Gaussian, at least for some parameter values. Figure 2, for example, displays the case $h=-1, \delta=1 / 2$, which compares well to the Gaussian

$$
z=-1+0.19 \exp \left(-\frac{x^{2}}{e^{2}}\right) .
$$

This is remarkable as we know that Gaussian bottoms lead to tidal conversion (Llewellyn Smith \& Young 2002), while the special ridge profile which this particular Gaussian resembles so closely, (4.15), does not.

Although the sensitive dependence to the exact shape of the topography requires further investigation, it seems that this is another expression of the ill-posed nature of hyperbolic BVPs, seen before in the internal wave context, e.g. in the streamfunction field's fractal dependence on a parabolic basin's scaled depth $d$ (Maas \& Lam 1995). In this context it appeared that linear fluid dynamical systems may display features 


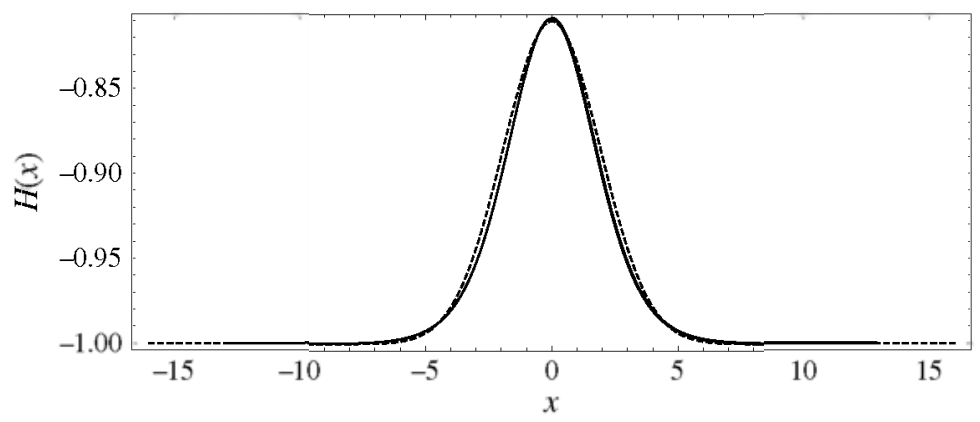

FIGURE 2. Ridge defined by inverse of (4.15) (solid), and Gaussian (4.16) (dashed).

that we normally associate with nonlinear dynamics, such as a sensitive dependence on domain shape (Maas 2005). However, it is worthwhile to note that this may also be a more general property of linear BVPs, as a similar sensitive dependence on topographic shape was recently found for linear surface waves, described by an elliptic (Laplace) equation (Howard \& Yu 2007).

For our ridge map, (4.11), the derivative reads

$$
f_{q}=\frac{1}{2}\left(1+\frac{\delta}{2 \cosh ^{2}(q / 2)}\right) .
$$

Hence, non-vanishing of the Jacobian is readily checked for ridges (having $\delta>0$ ). For deep trenches $(\delta<-2)$, however, this is no longer the case.

In the transformed plane, the barotropic solution for our particular tidal 'conversion' $\mathrm{BVP}$ is thus given by

$$
\psi=\frac{\zeta}{h}=\frac{z}{h}+\frac{\delta}{h} \frac{\sinh z}{\cosh z+\cosh x} .
$$

The solution is non-unique - we can add any number of channel internal modes - but there is no way one can infer the strength of these additional modes: there is no coupling. Clearly, also each of these channel internal modes is uncoupled to any of the other modes. The real-valued streamfunction (4.18), plotted in figure $3(a)$, is therefore also non-radiating. Including its harmonic time dependence, it consists simply of a blinking solution that decays away from the ridge. While its interpretation as barotropic mode is very obvious in the transformed plane, in the physical $(x, z)$-plane one would be inclined to interpret it as the combined barotropic-baroclinic response. Reiterating the discussion in $\S 2$, the reason is that in the physical plane the barotropic part of the streamfunction field is usually associated with a part strictly linear in $z$, so that the corresponding horizontal flow, given by its $z$-derivative, is depth-independent. Clearly, this is not the case for (4.18). Figure $3(b)$ shows the baroclinic $\psi^{\prime}$-field, obtained by subtracting the 'barotropic' streamfunction $\bar{\psi}=z / H(x)$, which was already displayed in the Introduction. This $\psi^{\prime}$-field solves (2.3), and vanishes correctly at the surface and bottom. It turns out that the $\psi^{\prime}$-field has a triple cell structure which is remarkably similar in form to the trapped tidal response computed, using quasi-linear advection by the barotropic tide, over a ridge of infinitesimal height (Maas \& Zimmerman 1989).

When use is made of the solution over the comparison topography (choosing $h=-d), \quad \psi_{c}=z / h$, the Green's function method should produce the combined 
(a)

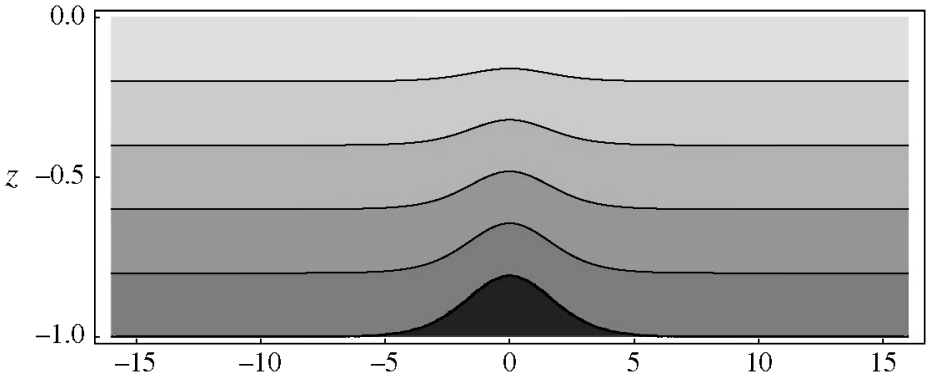

(b)

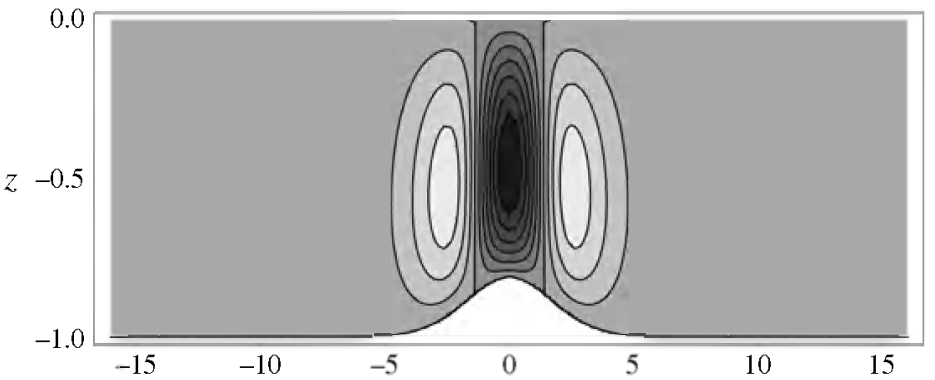

(c)

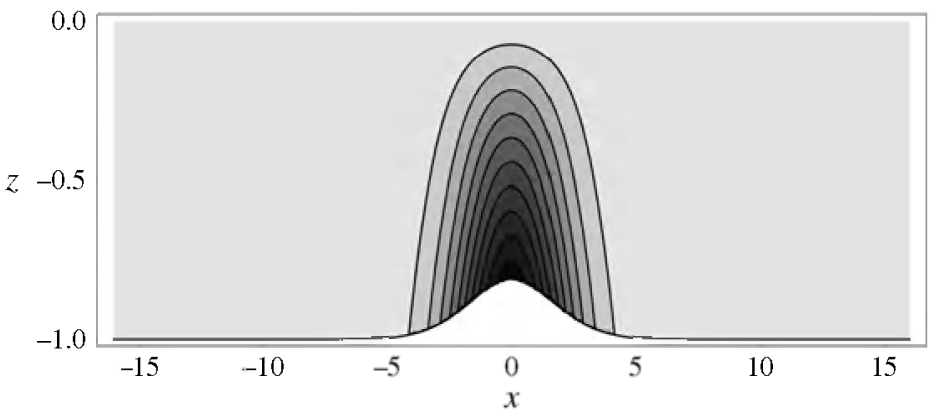

FIGURE 3. (a) Exact streamfunction field, $\psi$, (4.18), solving the internal tide generation problem over the ridge whose shape is defined by (4.15); contour interval: 0.2. (b) Baroclinic streamfunction field over a ridge obtained upon subtracting the 'barotropic' streamfunction, $\bar{\psi}=z / H(x)$, from the exact streamfunction (contour interval 0.00054 , from -0.00324 up). Here $h=-1$ and $\delta=1 / 2$. (c) Barotropic plus baroclinic response, $\tilde{\psi}$, obtained upon subtracting the streamfunction solution of a channel comparison topography, $\psi_{c}=z / h$, from the exact streamfunction (contour interval: 0.018).

barotropic-baroclinic field presented in figure $3(c)$. The non-vanishing of $\tilde{\psi}$ at the bottom is necessary to compensate for the apparent flow due to $\psi_{c}$ upon insertion of the ridge topography. $\tilde{\psi}=\psi-\psi_{c}$ still contains both a barotropic as well as a baroclinic component, not clearly separated from one another. This separation usually becomes evident when the solution separates into a part that is trapped at the ridge and a part that propagates away. Here, the only response is a trapped response. So this response would (again) be interpreted as a purely barotropic adjustment to the insertion of the ridge - in agreement with the interpretation in the transformed plane, even when the horizontal velocity is still depth-dependent. 


\subsection{Slope-shelf topography: $f(q)=q[1+\delta \tanh (q / 2)] / 2$}

When we choose $f(q)=q[1+\delta \tanh (q / 2)] / 2$ we obtain as new coordinate pair

$$
\begin{aligned}
& \xi(x, z)=x+\delta \frac{x \sinh x+z \sinh z}{\cosh z+\cosh x} . \\
& \zeta(x, z)=z+\delta \frac{z \sinh x+x \sinh z}{\cosh z+\cosh x} .
\end{aligned}
$$

Note that in comparison to (4.11), this mapping function, $f(q)$, differs by only one extra multiplicative factor $q$ in the second term. In the limit $x \rightarrow \pm \infty$, the new coordinates reduce to

$$
\begin{aligned}
& \xi \rightarrow x(1 \pm \delta), \\
& \zeta \rightarrow z(1 \pm \delta) .
\end{aligned}
$$

We require $\delta<1$, to prevent the bottom from protruding through the surface, $\zeta=0$. It turns out that, in order to prevent the Jacobian from vanishing, we actually have to impose the stricter requirement $\delta<\delta_{c} \approx 0.83357$. The latter is the value for $\delta$ at which the map's derivative $f^{\prime}(q)$ first vanishes. This occurs at the derivative's minimum, obtained where $f^{\prime \prime}\left(q_{*}\right)=0$. With $p \equiv q_{*} / 2$, this is found when

$$
p \tanh p=1,
$$

so that, from $f^{\prime}\left(q_{*}\right)=0$, we find $\delta_{c}=1 / p$.

The bottom $H(x: h, \delta)$, that depends parametrically on $h$ and $\delta$, is now determined implicitly by the solution of

$$
h=z+\delta \frac{z \sinh x+x \sinh z}{\cosh z+\cosh x} .
$$

Far away from the sloping region, for $x \rightarrow \pm \infty$, (4.24) yields topography $z=H(x)$ that has asymptotic depths $H_{ \pm \infty}=h /(1 \pm \delta)$. This tells us that $h$, obtained from

$$
\frac{1}{h}=\frac{1}{2}\left(\frac{1}{H_{\infty}}+\frac{1}{H_{-\infty}}\right),
$$

is the harmonic mean of the asymptotic depths in the physical plane. The second parameter,

$$
\delta=\frac{H_{-\infty}-H_{\infty}}{\boldsymbol{H}_{-\infty}+\boldsymbol{H}_{\infty}},
$$

represents the relative depth contrast, the difference in asymptotic depths divided by their sum. The origin, $x=0$, is located where the depth equals the mean depth, $z=h$. In figure 4 we have taken $h=-1$ and $\delta=1 / 2$, equivalent to $H_{-\infty}=-2$ and $H_{\infty}=$ $-2 / 3$. The bottom is shown together with the 'baroclinic' solution, $\psi$ ' $=\psi-z / H(x)$.

There are other shelf-slope topographic shapes having the same asymptotic depths, e.g. the piecewise linear topography (Baines 1973; Craig 1987), which do convert the surface tide into internal tides. But even when the peculiar topographic shape of figure 4 is frozen, and a different tidal frequency is adopted - implying a different characteristic slope and different depth scale, conversion of the surface tide will take place as usual. This might suggest that the absence of tidal conversion for the original frequency and topography of figure 4 is an anomaly. But, given this topography's unscaled asymptotic depths, this new frequency leads to another "non-converting topography' which is, grossly speaking, of similar shape, differing only in detail from 
(a)

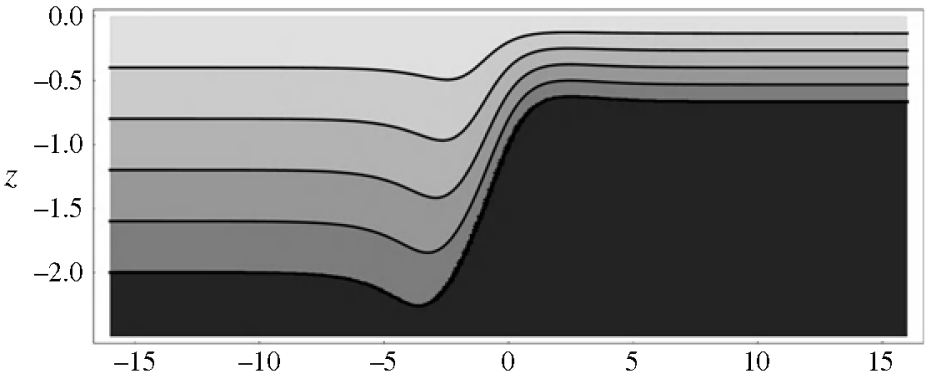

(b)

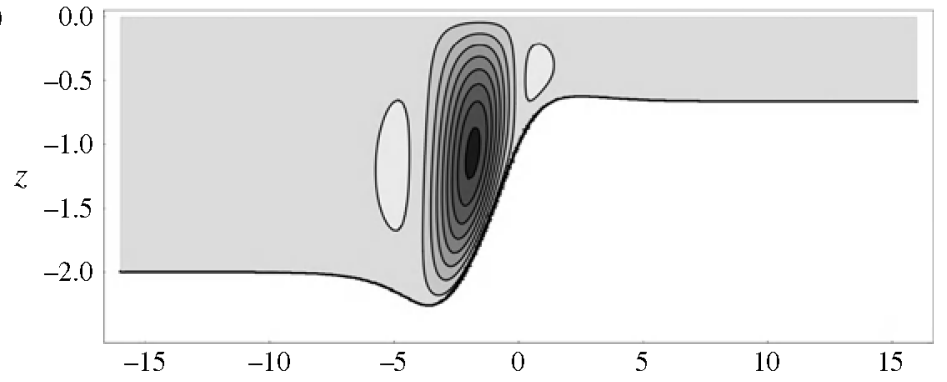

(c)

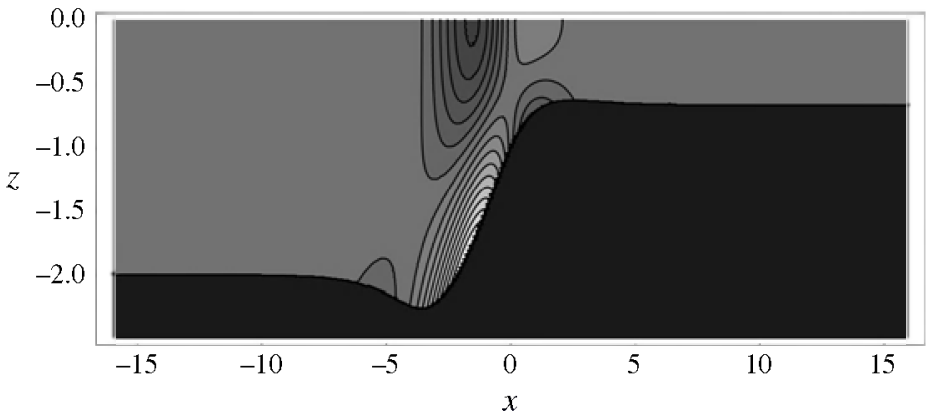

FIGURE 4. (a) Exact streamfunction field, $\psi$, (4.18), solving the internal tide generation problem over the slope-shelf defined by (4.24); contour interval: 0.2. (b) Baroclinic streamfunction field over a slope-shelf obtained upon subtracting a 'barotropic' streamfunction, $\bar{\psi}=z / H(x)$, from the exact streamfunction (contour interval 0.01 , from -0.075 up). Here $h=-1$ and $\delta=1 / 2$. (c) Corresponding baroclinic horizontal velocity field, $u^{\prime}=-\psi_{z}^{\prime}$ (contour interval 0.02 , from $-0.11 \mathrm{up}$ ).

the original. It illustrates how to read the title of this paper: given a stratification $N$ and tidal frequency $\omega$, for any set of shelf and ocean depths there exists a particular slope-shelf bottom shape lacking tidal conversion. This statement should be slightly rephrased for other geometries. For the ridge in the previous example, for instance, 'shelf depth' should be replaced by 'ridge height'.

As a sequel to the present analytical study, a numerical internal tide generation model (Gerkema 2002) is being used to examine the internal tide generation (or, rather, the absence thereof) for the slope and ridge topographies and the precise forcing frequencies that correspond to the situation considered in this paper. Preliminary results are promising, but the conversion rates, although extremely small, are not exactly zero (Theo Gerkema, personal communication). Presumably, this is just the result of numerical inaccuracies, but further study is required to corroborate this. 
4.4. Near-sinusoidal topography: $f(q)=(q+\delta \cos q) / 2$

The choice $f(q)=(q+\delta \cos q) / 2$ leads to a transformed coordinate pair

$$
\begin{aligned}
& \xi=x+\delta \cos x \cos z, \\
& \zeta=z-\delta \sin x \sin z .
\end{aligned}
$$

The bottom of the channel, $z=H(x)$, is mapped onto the constant depth, $\zeta=h$, and is obtained by solving

$$
h=z-\delta \sin x \sin z .
$$

This leads to a bottom, $x=\bar{H}(z)$ :

$$
x=\bar{H}(z)=\arcsin \left(\frac{z-h}{\delta \sin z}\right) .
$$

Extracting the inverse description from (4.29), $z=H(x)$, is in fact Kepler's problem (Watson 1966; Howard \& Yu 2007). For $\delta<1$ it yields

$$
z=H(x)=h+\sum_{n=1}^{\infty} \frac{2}{n} \mathbf{J}_{n}(n \delta \sin x) \sin (n h),
$$

in terms of $n$ th-order Bessel functions $\mathbf{J}_{k}(\cdot)$. Since $H(x+2 m \pi)=H(x), m \in \mathbb{Z}$, the bottom is $2 \pi$-periodic. It offers an example in which the BVP can be solved for a topography that does not revert to a constant depth. When $\delta \rightarrow 0$, the bottom becomes approximately sinusoidal:

$$
z=H(x) \approx h+\delta \sin x \sin h,
$$

as can be found from Bessel function near-field expansions in (4.31), or from (4.29), by simply using the zeroth-order result, $z=h$, in its $O(\delta)$-term. Depth scale $d$ sets the average depth, and we thus find $h=-d$.

Bessel functions of integer order $n>0$ vanish for zero argument, so that $H(m \pi)=h$, for $m \in \mathbb{N}$. Thus in general, depth scale $d$ at these $x$-positions defines $h$. The second parameter, $\delta$, now relates to the slope of the topography at these positions, $H^{\prime}(m \pi)$. This follows from the Bessel function derivative

$$
\frac{\mathrm{d}}{\mathrm{d} x} \mathbf{J}_{n}(n \delta \sin x)=\frac{1}{2} n \delta \cos x\left[\mathbf{J}_{n-1}(n \delta \sin x)-\mathbf{J}_{n+1}(n \delta \sin x)\right]
$$

and the fact that only the zeroth-order Bessel function is non-vanishing at the origin, $\mathbf{J}_{0}(0)=1$,

$$
H^{\prime}(m \pi)=(-1)^{m} \delta \sin h, \quad m \in \mathbb{N} .
$$

Non-vanishing of the Jacobian and subcriticality of the bottom slope $\left(\left|H^{\prime}\right|<1\right)$ is in this case guaranteed for any depth $h$ whenever $|\delta|<1$. The total streamfunction response, plotted in the Cartesian frame of reference, is given in figure $5(a)$. The baroclinic response is shown in figure $5(b)$ and is prominently present only over the valleys.

A referee pointed to the possibility of using this example to construct a topography having a finite support. This is obtained by employing the (related) piecewise defined function

$$
f(q)=\frac{1}{2(1-\delta)}(q+\delta \sin q), \quad|q|<\pi,
$$


(a)

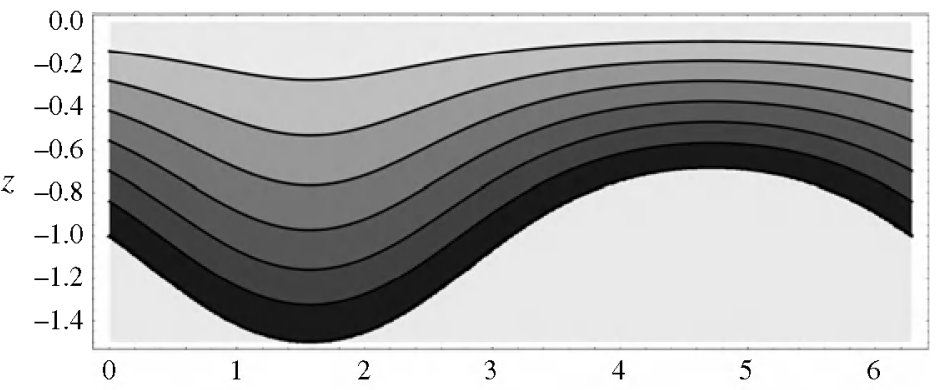

(b)

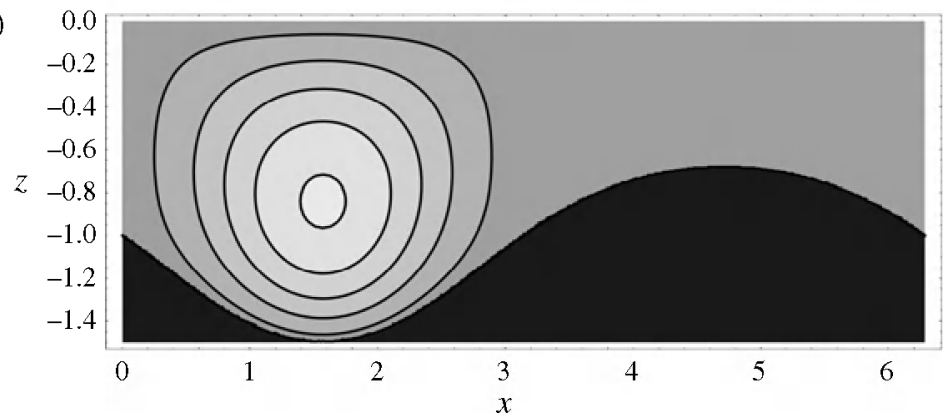

FIGURE 5. (a) Total streamfunction field $\psi$ (contour interval 0.14 ) and $(b)$ baroclinic streamfunction field $\psi^{\prime}$ (contour interval 0.01) for near-sinusoidal topography (4.31) using $h=-1 ; \delta=1 / 2$, shown over one wavelength.

$$
f(q)=\frac{1}{2}\left(q \pm \frac{\delta \pi}{1-\delta}\right), \quad \pm q \geqslant \pi .
$$

This choice guarantees that $f$ and its first and second derivatives are continuous at $|q|=\pi$. It leads to a bottom shape consisting of a ridge that extends over a finite region, $|x| \leqslant X \equiv 1+\pi$, outside of which the resulting topography is completely flat (bottom at $z=-1$ ). This ridge topography (of height controlled by parameter $\delta$ ) is thus similar to that treated in $\S 4.2$. But in this case, the triple-cell, trapped response is strictly confined to the ridge region, $|x| \leqslant X$, which should therefore be directly comparable to results obtained over ridges of finite support studied before (Pétrélis et al. 2006).

\section{Discussion}

The curious absence of tidal conversion, found here, seems to be a commonplace phenomenon in the classical internal tide generation model that employs a simple back-and-forth barotropic flow over topography in a uniformly stratified, rigid-lid fluid. In fact, it makes one wonder what the impact of this feature is on topographies that do convert the barotropic tide into internal tides, especially since the 'distance' between converting and non-converting topographies (measured in some appropriate norm) might become very small, as illustrated in figure 2 . The latter example in fact suggests that, using (4.15) as comparison topography, transformation (4.13) maps a converting topography, as the Gaussian in this figure, onto a nearly uniform-depth channel, where it then manifests itself as infinitesimal topographic irregularity. This would allow the use of perturbation techniques, probably giving accurate results on the 
conversion in the transformed plane, which can then subsequently be expressed in the Cartesian frame.

It is interesting to speculate whether the absence of tidal conversion over certain bottom shapes may actually steer the evolution of bottom profiles. The ability of internal tides to shape continental slopes was previously addressed by Cacchione, Pratson \& Ogston (2002). To see how this works here, suppose the ocean bottom consists of a combination of rock and movable sediments. Now further suppose that the initial bottom profile that one starts out with gives rise to internal tide generation. Would it be possible for the barotropic and baroclinic motions to lead to internal tide generation shut-down? This might occur when the currents reshuffle the sediments so as to create a bottom profile (set by the rocky bottom's mean depth and depth-contrast) for which tidal conversion is absent; a topographic shape that, from the perspective of the barotropic tide, 'just feels right'. To rephrase this: Are non-converting topographies 'attracting' in a dynamical sedimentological sense?

The most interesting question is whether topographies lacking tidal conversion actually exist. The analytical derivation in $\S 2$ seems to show that the absence of tidal conversion is definitely a property of the simple internal tide generation model, as succinctly posed in e.g. Baines (1982). Yet, there are arguments to question the nature and perhaps validity of this model, all centring on the meaning and implementation of the radiation condition, and on the division between a trapped barotropic versus propagating baroclinic response.

For instance, the reason why tidal conversion is absent in this simple model appears to be due to pressure variations being exactly out-of-phase (in quadrature) with velocity perturbations. But phase shifts between the two can be realized in several ways, both inviscidly as well as by means of viscous processes, so that the absence of tidal conversion seems unrealistic. Inviscidly, phase shifts occur when the surface tide is no longer modelled as a 'frozen' hydraulic flow, but instead is allowed to display some scattering effects due to (partial) reflection by depth inhomogeneities (Mei 1989) or by loss of some of its energy to the internal tide. Including viscous or frictional effects, the problem becomes singularly perturbed, since its highest derivative is then preceded by a small parameter - the eddy viscosity. This has the remarkable property that the phase shift, produced by this viscous process, leads to a result which, in the limit that viscosity is decreased to zero, differs from that obtained when viscous effects are considered absent ab initio (Ou \& Bennett 1979; Maas, Zimmerman \& Temme 1987). It is quite likely that the internal wave radiation condition, which involves 'pushing poles' away from the real axis by an infinitesimal amount, rests on a similar underlying limiting process, which, while rendering the presence of viscosity invisible, is yet important.

Also, in the small-topography limit, $\delta \ll 1$, Llewellyn Smith \& Young (2002) showed that tidal conversion is given by the sum of the squared topographic spectral function, sampled at discrete wavenumbers, set by the ocean depth. This suggests that tidal conversion can vanish only when the topographic spectrum has zeros precisely at these wavenumbers (Oliver Bühler, personal communication), which is an unlikely property for the topographic shapes discussed in this paper.

Counter to this argument, however, is the already quoted fact that, depending on the precise parameter setting, numerical models, necessarily having some artificial damping, do seem able to show both the presence or (near) absence of tidal conversion. This suggests that the absence of tidal conversion is real after all (see also Griffiths \& Grimshaw 2007). It also suggests that simplified numerical internal tide generation models are able to produce the required shift in phase between pressure and velocity 
fields. While the origin of this phase shift needs further scrutiny, its magnitude is probably important in determining the relative contributions to the trapped versus propagating fields.

Howard \& Yu (2007) observed that the surface wave problem in a channel of constant depth can be solved purely in terms of propagating modes, without the necessity of invoking the family of evanescent modes, trapped at the end walls. In their case, this changed when the bottom was no longer flat. Here we seem to find the opposite: we have a large family of bottom profiles for which the evanescent modes suffice to solve the internal tide 'generation and scattering' problems, without the need to invoke propagating modes. Clearly, this restriction was produced by the requirement that the topography could once again be mapped onto a constant-depth channel. Perhaps, therefore, the present work can be combined with the continued fraction methods developed in Howard \& Yu (2007) and Yu \& Howard (2010) to see how the propagating modes participate for more general topographic shapes and how these can be computed analytically. Possibly this approach may give insight also in the question of how to circumvent points or lines where the Jacobian vanishes, so as to be able to apply the method to supercritically sloping topography as well.

Discussions with U. Harlander, T. Gerkema and $\mathrm{H}$. van Haren and comments by three referees are gratefully acknowledged. I thank T. Gerkema for performing initial numerical computations and $\mathrm{R}$. Camassa for pointing me towards the recent literature on surface waves. This work was completed while the author was on sabbatical leave at the University of Porto; hospitality offered there by J. da Silva is gratefully acknowledged.

\section{Appendix. Internal tide generation model}

In a Cartesian $(x, z)$-frame of reference, the Boussinesq equations governing linear, inviscid, monochromatic waves of frequency $\omega>0, \propto \exp (-\mathrm{i} \omega t)$, propagating in a uniformly stratified, two-dimensional channel, confined between bottom $(z=H(x)<0)$ and rigid-lid surface $(z=0)$, read (Vlasenko et al. 2005)

$$
\begin{gathered}
-\mathrm{i} \omega u=-p_{x}, \\
-\mathbf{i} \omega w=-p_{z}+b, \\
-\mathbf{i} \omega b+w N^{2}=0, \\
u_{x}+w_{z}=0 .
\end{gathered}
$$

Here the subscript-derivative convention is employed. Dropping the common timedependence, perturbations are represented by velocity components $(u, w)$, in horizontal and vertical $(x, z)$-directions respectively, buoyancy $b=-g \rho^{\prime} / \rho_{\text {; }}$ and by pressure $p$ (divided by $\rho$ ). The density field thus consists of uniform reference density $\rho_{*}$, the stationary horizontally uniform stratification $\rho_{0}(z)$, and perturbation density $\rho^{\prime}(x, z) \exp (-i \omega t)$. Buoyancy frequency $N$, assumed constant here, is defined through $N^{2}=-g \rho_{*}^{-1} \mathrm{~d} \rho_{0} / \mathrm{d} z$.

The continuity equation can be used to introduce streamfunction $\psi$, where $u=-\psi_{z}$ and $w=\psi_{x}$. Using also the buoyancy equation to eliminate buoyancy from the vertical momentum equation, we obtain

$$
\begin{gathered}
\omega \psi_{z}=\mathrm{i} p_{x} \\
\left(N^{2}-\omega^{2}\right) \psi_{x}=\mathrm{i} \omega p_{z} .
\end{gathered}
$$


For the dependent and independent variables, we will employ scales, indicated by brackets [-], as follows: $[t, x, z, \psi, p]=\left(\omega^{-1}, L, L\left(N^{2} / \omega^{2}-1\right)^{-1 / 2}, Q_{*},-\mathrm{i} Q_{*} \omega\left(N^{2} /\right.\right.$ $\left.\omega^{2}-1\right)^{1 / 2}$, where $L$ is a typical horizontal scale of the topography, $Q$ a transport scale (e.g. the barotropic transport, when tidal conversion is considered) and where $\omega<N$ is assumed, rendering these scales real and delineating the internal wave frequency domain. Note that the $-\mathbf{i}$ factor in the pressure scaling indicates that in this inviscid problem the pressure always lags behind the streamfunction field by a quarter cycle. This out-of-phase relationship implies the vanishing of an outward-directed baroclinic energy flux when both computed fields are real (Sandstrom 1976), as in the absence of conversion studied here. Ocean depth scale $D$ is scaled by the same factor as the vertical coordinate and now reads

$$
d \equiv \frac{D}{L}\left(\frac{N^{2}}{\omega^{2}}-1\right)^{1 / 2} .
$$

Rotational effects are excluded here but on an $f$-plane these can, without loss of generality, be absorbed in redefining scales and dimensionless depth $d$. When, moreover, variations in along-isobath $y$-direction are absent, and the waves propagate perpendicular to the bathymetry $\left(\partial_{y}=0\right)$, the above $z$ - and $p$-scales are rotationally modified by multiplying them by $\left(1-f^{2} / \omega^{2}\right)^{1 / 2}$, while depth $d$, (A 7), is divided by this factor. Requiring these scales to be real restricts the internal wave frequency interval, as usual, to $f<\omega<N$.

Inserting $\phi=[\phi] \phi^{\prime}$ into (A 6), where $\phi$ represents any of the dependent or independent variables, using the above scales and then dropping primes, these equations simplify to

$$
\begin{aligned}
& \psi_{z}=p_{x} . \\
& \psi_{x}=p_{z} .
\end{aligned}
$$

From this we see that streamfunction and pressure are two complementary functions, both satisfying a spatial hyperbolic equation,

$$
\psi_{x x}-\psi_{z z}=0,
$$

and

$$
p_{x x}-p_{z z}=0 .
$$

Equations (A 8) and (A 9) are analogous to those derived for the real, $\xi$, and imaginary, $\eta$, parts of a complex quantity $\alpha$ :

$$
\alpha=\xi+\mathrm{i} \eta=F(a) .
$$

which is an arbitrary function $F$ of complex coordinate $a=x+\mathrm{i} y$. To find these equations, take the $x$ - and $y$-derivative of (A12),

$$
\begin{aligned}
& \xi_{x}+\mathrm{i} \eta_{x}=F_{a}, \\
& \xi_{y}+\mathrm{i} \eta_{y}=\mathrm{i} F_{a} .
\end{aligned}
$$

Adding $\mathrm{i}$ times the second to the first equation, and requiring separate vanishing of real and imaginary parts, leads to the Cauchy-Riemann equations:

$$
\begin{gathered}
\xi_{x}=\eta_{y}, \\
\xi_{y}=-\eta_{x} .
\end{gathered}
$$

By cross-differentiation and addition one observes that both $\xi$ and $\eta$ satisfy the (elliptic) Laplace equation, $\Delta(\xi, \eta)=0$, where $\Delta \equiv \partial_{x x}+\partial_{y y}$. Because of the analogy 
between (A 8) and (A 9) to (A 15) and (A 16) we will say that $\psi$ and $p$ satisfy hyperbolic Cauchy-Riemann equations (see Motter \& Rosa 1998 for a discussion).

\section{REFERENCES}

BAines, P. G. 1973 The generation of internal tides by flat-bump topography. Deep-Sea Res. 20 (2), 179-205.

Baines, P. G. 1982 On internal tide generation models. Deep-Sea Res. 29 (3), 307-338.

Balmforth, N. J., IERley, G. R. \& Young, W. R. 2002 Tidal conversion by subcritical topography. J. Phys. Oceanogr. 32 (10), 2900-2914.

BÜHLer. O. \& Holmes CeRFon. M. 2011 Scattering of internal tides by random topography. J. Fluid Mech. 638. 5-26.

Cacchione. D. A., Pratson. L. F. \& Ogston. A. S. 2002 The shaping of continental slopes by internal tides. Science 296. 724-727.

Craig, P. D. 1987 Solutions for internal tidal generation over coastal topography. J. Mar. Res. 45, 83-105.

Garrett, C. \& Gerkema, T. 2007 On the body-force term in internal-tide generation. J. Phys. Oceanogr. 37 (8), 2172.

GerkemA. T. 2002 Application of an internal tide generation model to baroclinic spring-neap cycles. J. Geophys. Res. 107 (C9), 3124-3131.

Gerkema. T. 2011 Comment on 'Internal-tide energy over topography' by Kelly et al. J. Geophys. Res. (in press).

Griffiths, S. D. \& GRimshaw, R. H. J. 2007 Internal tide generation at the continental shelf modeled using a modal decomposition: two-dimensional results. J. Phys. Oceanogr. 37. 428-451.

Howard, L. N. \& YU, J. 2007 Normal modes of a rectangular tank with corrugated bottom. J. Fluid Mech. 593, 209-234.

Llewellyn Smith, S. G. \& Young, W. R. 2002 Conversion of the barotropic tide. J. Phys. Oceanogr. 32 (2). 1554-1566.

MAAS. L. R. M. 2005 Wave attractors: linear yet nonlinear. Intl J. Bifurcation Chaos 15, 2757-2782.

MAas. L. R. M. 2009 Exact analytic self-similar solution of a wave attractor field. Physica D 238 (5). $502-505$.

MAAS, L. R. M. \& LAM. F. P. A. 1995 Geometric focusing of internal waves. J. Fluid Mech. 300. 1-41.

MaAs, L. R. M. \& Zimmerman, J. T. F. 1989 Tide-topography interactions in a stratified shelf sea. Part II. Bottom-trapped internal tides and baroclinic residual currents. Geophys. Astrophys. Fluid Dyn. 45. $37-69$.

MaAs, L. R. M., Zimmerman. J. T. F. \& Temme, N. M. 1987 On the exact shape of the horizontal profile of a topographically rectified tidal flow. Geophys. Astrophys. Fluid Dyn. 38, 105-129.

MEI, C. C. 1989 The Applied Dynamics of Ocean Surface Waves. World Scientific.

Motter. A. E. \& Rosa. M. A. F. 1998 Hyperbolic calculus. Advances in Applied Clifford Algebras 8. $109-128$.

MülLER, P. \& LiU, X. 2000 Scattering of internal waves at finite topography in two dimensions. Part I. Theory and case studies. J. Phys. Oceanogr. 30 (3). 532.

Ou, H. W. \& BenNeTT, J. R. 1979 A theory of the mean flow driven by long internal waves in a rotating basin, with application to Lake Kinneret. J. Phys. Oceanogr. 6, 1112-1125.

OU, H. W. \& MAAS, L. R. M. 1986 Tidal-induced buoyancy flux and mean transverse circulation. Cont. Shelf Res. 5, 611-628.

Pétrélis, F. Llewellyn Smith, S. \& Young, W. R. 2006 Tidal conversion at a submarine ridge. J. Phys. Oceanogr. 36 (6), 1053-1071.

SANDSTROM. H. 1976 On topographic generation and coupling of internal waves. Geophys. Astrophys. Fluid Dyn. 7, 231-270.

St Laurent, L.. Stringer. S., Garrett, C. \& Perrault-Joncas, D. 2003 The generation of internal tides at abrupt topography. Deep-Sea Res. (I) 50 (8). 987-1003.

Vlasenko, V., Stashchuk, N. \& HutTer, K. 2005 Baroclinic Tides: Theoretical Modeling and Observational Evidence. Cambridge University Press.

Watson, G. N. 1966 A Treatise on the Theory of Bessel Functions, 2nd edn. Cambridge University Press. 
Wunsch, C. 1968 On the propagation of internal waves up a slope. Deep-Sea Res. 15, 251-258.

Wunsch, C. 1971 Note on some Reynolds stress effects of internal waves on slopes. Deep-Sea Res. 18 (6), $583-591$.

YU, J. \& HowaRD, L. N. 2010 On higher order Bragg resonance of water waves by bottom corrugations. J. Fluid Mech. 659, 484-504. 